\title{
Sinus rate in acute myocardial infarction
}

\author{
R. M. Norris, C. J. Mercer, and S. E. Yeates \\ -From Green Lane Hospital, Auckland, New Zealand
}

The clinical course of sinus bradycardia and sinus tachycardia has been studied in 735 patients - with acute myocardial infarction admitted to a coronary care unit. Sinus bradycardia was considered to have occurred when two or more records showed sinus rhythm with a rate below 60 a minute, and sinus tachycardia when a sinus rate over 100 a minute occurred in two or more records. Hospital mortality of patients having sinus bradycardia was significantly lower (6\%) and of those with sinus tachycardia was significantly higher (26\%) than of those who had neither bradycardia nor tachycardia (15\%). Major ventricular arrhythmias were no more common in patients having sinus bradycardia and when they did occur did not usually do so at the time "that the bradycardia was present. Sinus bradycardia was more common in cases of posterior and subendocardial infarction without radiological evidence of cardiac failure, while sinus tachycardia was frequent in patients with anterior transmural infarction complicated by heart failure. The benign course of sinus bradycardia is surprising because experimental evidence suggests that slow heart rates after infarction favour the occurrence of ventricular arrhythmias. Further work 'is necessary to correlate heart rate with prognosis, particularly in patients seen at the onset of infarction, and before admission to a coronary care unit.

Observations of patients in coronary care units has shown that wide variations in sinus rate occur in patients who have suffered acute - myocardial infarction. Sinus tachycardia has been found to be associated with a high mortality, while sinus bradycardia has in general reen found to be benign and not associated with serious complications (Lawrie et al., 1967; Jewitt et al., 1967; Chapman, 1971). Experiments on dogs (Han et al., I966a, b; Han, 1969), however, have shown that slow heart rates after infarction favour the occur" rence of ventricular arrhythmias, and on this account aggressive treatment of bradycardia Yi favoured by some (Lown et al., 1967; Gregory and Grace, 1968). Moreover, as sinus bradycardia occurs most commonly at the onset of infarction before most patients come under medical attention, it has been - suggested that sinus bradycardia occurring early after the onset may have a more serious Nignificance than when the arrhythmia occurs later (Adgey et al., 1968, 197 I ; Stock, I97 I).

The purpose of this paper is to amplify previous observations (Norris, 1969a; Norris and Mercer, 1970) on the benign course of sinus - bradycardia as seen in a coronary care unit, to draw attention to the conflict between the

Received 2 I December I97I. clinical and experimental evidence, and to suggest that further studies on the association of bradyarrhythmias with ventricular fibrillation are necessary under a wider variety of clinical conditions.

\section{Patients and methods}

Patients were all those admitted to the fourbedded coronary-care unit at Green Lane Hospital over three years. Myocardial infarction was considered to have occurred if two or more of the following criteria were satisfied: (I) Characteristic clinical presentation; (2) pathological $Q$ waves, ST elevation, or $T$ wave inversion in the electrocardiogram with evolutionary changes; and (3) rise in serum aspartate aminotransferase (SGOT) to over 40 units $/ \mathrm{ml}$. Patients over 70 years of age were in general excluded because of shortage of beds, 53 per cent of patients were admitted within six hours of onset of the most severe chest pain, and stay in the unit was three days on average.

Ten-second electrocardiograms were made hourly or whenever an arrhythmia occurred, and clinical data with details of arrhythmias were recorded on punch cards (International Business Machines). Sinus bradycardia was considered to have occurred if two or more records showed sinus rhythm with a rate below 60 a minute. In order to study possible effects or associations of sinus bradycardia with prognosis and with other arrhythmias, the findings were compared with 
those in patients who had sinus tachycardia (two or more records with a sinus rate over IOO a minute), those who had both sinus bradycardia and sinus tachycardia and those who had neither of these arrhythmias. Cases of sinus bradycardia were also examined in relation to the lowest recorded heart rate, the time of occurrence after admission to the coronary care unit, and the site of infarction and degree of cardiac failure assessed from the electrocardiogram and chest $x$-ray.

\section{Results}

During the three years, 1223 patients were admitted to the coronary care unit and, of these, 735 had acute myocardial infarction according to the above criteria, and were unselected cases, not referred from other hospitals. Of these 735 patients, $152(21 \%)$ had sinus bradycardia, $267(36 \%)$ had sinus tachycardia, 4I (6\%) had both sinus bradycardia and tachycardia, and $275(37 \%)$ had neither arrhythmia (Table I). Hospital mortality was significantly lower $\left(6 \% ; \chi^{2}=8 \cdot 1, \mathrm{P}<0.01\right)$ in patients who had sinus bradycardia, and significantly greater $\left(26 \% ; \chi^{2}=9.9, \mathrm{P}<0.01\right)$ in those who had sinus tachycardia, when these groups of patients were compared with those who had neither (mortality $15 \%$ ).

The incidence of ventricular fibrillation and major ventricular arrhythmias in the same groups of patients is also shown in Table $I$. Major ventricular arrhythmias were classified as salvoes of two or more ventricular ectopic beats, ventricular ectopic beats showing the $R$ on $T$ phenomenon, ventricular tachycardia, and ventricular fibrillation. We have found the first three of these arrhythmias to be associated with a significantly increased incidence of ventricular fibrillation (R. M. Norris, 1968, unpublished observations). There was no significant difference in the incidence of ventricular arrhythmias among any of the groups, though there was a tendency for patients with sinus tachycardia to have a higher incidence of major ventricular arrhythmias and ventricular fibrillation. Of the II patients who had both sinus bradycardia and ventricular fibrillation, 7 had ventricular fibrillation while they were being observed in the coronary care unit. In all of these patients ventricular fibrillation occurred at a time when sinus bradycardia was not present. Moreover, ventricular fibrillation and major ventricular arrhythmias were no more common when sinus bradycardia occurred within one hour of admission (approximately $50 \%$ of cases) than when it occurred later.

Further analysis of the cases of sinus bradycardia showed that in approximately 60 per cent of cases the lowest recorded heart rate
TABLE I Sinus tachycardia and sinus bradycardia complicating acute myocardial infarction - incidence, mortality, and association with major ventricular arrhythmias

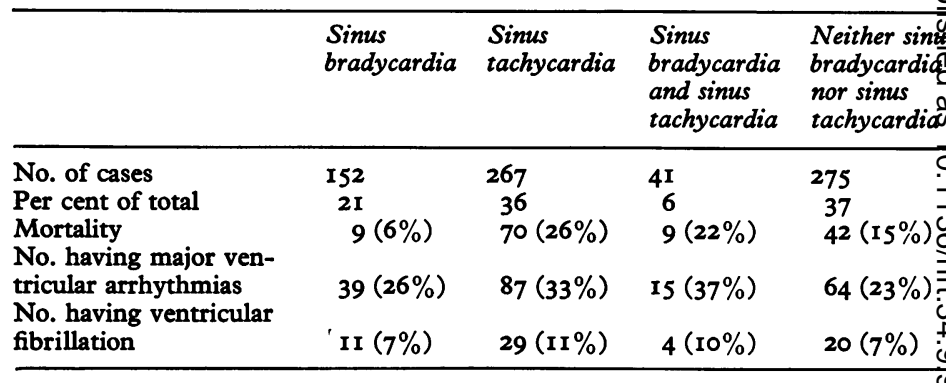

was between 50 and 60 a minute, in 30 per cent the rate was 40 to 50 a minute, while in Io per cent of cases it was less than 40 a minute, often with alternation between sinus and nodal pacemakers. Patients in the latter group usually had an abrupt onset of bradycardia accompanied by faintness, pallor, and hypotension. These attacks responded promptly to intravenous atropine 0.3 to 0.6 $\mathrm{mg}$, but in several cases when there was an inadvertent delay in giving atropine there was a spontaneous improvement in heart rate. Of 13 patients in whom sinus bradycardia at a rate of less than 40 a minute was observed, only one had major ventricular arrhythmias as described above, and none had ventricular fibrillation. The possibility that some cases of sinus bradycardia were due to therapy was also considered, and in 8 cases it was possible that alprenolol, which was the subject of a clinical trial (Briant and Norris, 1970), was the cause of the arrhythmia. In some other patients morphine may have caused the bradycardia, particularly when vomiting occurred after giving the drug.

The relation of sinus bradycardia and sinus tachycardia to the site and extent of infarction

TABLE 2 Relation between site and extent of infarction and occurrence of sinus bradycardia and tachycardia

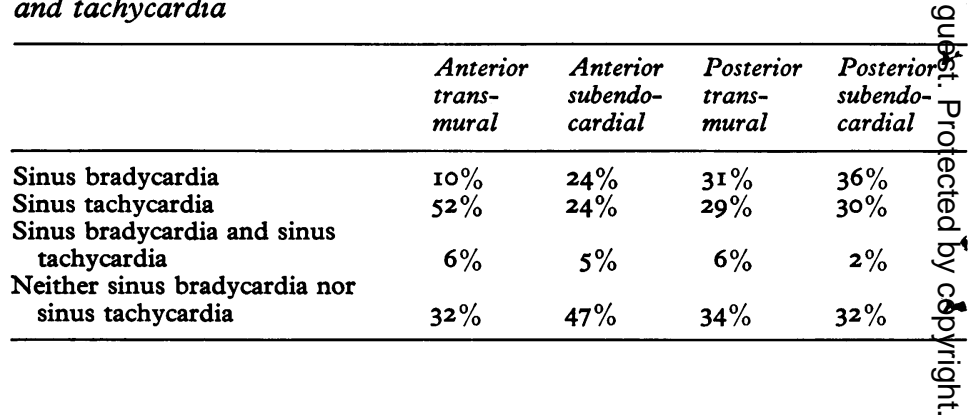


TABLE 3 Relation between sinus bradycardia and tachycardia and occurrence of radiological pulmonary oedema

\begin{tabular}{lcc}
\hline & $\begin{array}{l}\text { No. of } \\
\text { cases }\end{array}$ & $\begin{array}{l}\text { No. having } \\
\text { oedema }\end{array}$ \\
\hline $\begin{array}{l}\text { Sinus bradycardia } \\
\text { Sinus tachycardia }\end{array}$ & 58 & $7(12 \%)$ \\
$\begin{array}{l}\text { Sinus bradycardia and sinus } \\
\text { tachycardia }\end{array}$ & 115 & $53(46 \%)$ \\
$\begin{array}{l}\text { Neither sinus bradycardia } \\
\text { nor sinus tachycardia }\end{array}$ & 108 & $7(37 \%)$ \\
\hline
\end{tabular}

-

and the degree of cardiac failure is shown in Tables 2 and 3. Sinus bradycardia was commoner in posterior infarcts, and was also more frequent in subendocardial infarcts (ST and - $T$ wave changes only) than in transmural infarcts (infarction with pathological $Q$ waves), ,while sinus tachycardia occurred usually in cases of anterior transmural infarction. Table 3 refers to a group of 300 patients in whom the presence of pulmonary oedema in a chest $x$-ray taken on admission to hospital was used as a factor in constructing a coronary prognostic index (Norris, Brandt, and Lee, I969). Pulmonary oedema was present on admission in 46 per cent of these patients who had sinus tachycardia, but in only 12 per cent of those who had sinus bradycardia.

\section{Discussion}

The present results confirm previous reports (Lawrie et al., I967; Jewitt et al., I967; Chapman, I97I) that sinus bradycardia as seen in a coronary care unit is a benign arrhythmia which tends to be associated with small myo-

- cardial infarcts and a low incidence of cardiac failure. Its association mainly with posterior infarction, and its sensitivity to atropine, suggest that stimulation of vagal nerve endings in the posterior part of the atrioventricular groove may be the mechanism (James, I968), though the release of depressor substances ' such as adenosine (James, I965) may be responsible in some cases. The high incidence $(36 \%)$ and mortality $(26 \%)$ of patients with sinus tachycardia also confirm previous work (Lawrie et al., I967; Jewitt et al., 1967; Chapman, I97I), and the association of sinus tachycardia with large anterior infarcts and radio-

- logical evidence of pulmonary oedema suggests compensation by a damaged heart for a reduction in stroke volume.

The lack of association between sinus - bradycardia and serious ventricular arrhythmias has been reported less frequently. This Inding is surprising and worthy of comment, because of the experimental evidence that slow heart rates favour the occurrence of arrhythmias due to re-entry or spontaneous discharge of excitable myocardial cells (Han et al., I966a, b; Han, 1969), and the clinical evidence that 'over driving' the heart at a faster rate can suppress ventricular arrhythmias (Sowton, Leatham, and Carson, I964). The mechanisms of asynchronous repolarization and spontaneous depolarization of myocardial cells which have been described would presumably also operate in bradyarrhythmia due to atrioventricular block. We have found previously, however, that ventricular fibrillation is uncommon in the course of heart block complicating posterior infarction (Norris, I969b), while in heart block due to anterior infarction, cardiac arrest occurs not in ventricular fibrillation but in asystole (Norris, I969b; Norris and Croxson, I970). The reason for this apparent conflict of experimental and clinical evidence is not clear.

The possibility exists that sinus bradycardia at the very onset of myocardial infarction might lead to ventricular arrhythmias, while bradycardia later in the course of infarction may be more benign. That bradycardia is commoner at the onset is shown by the studies of Pantridge and his colleagues (Adgey et al., I968, I97I). They have shown that the incidence of sinus bradycardia in patients seen within the first hour after the onset of infarction is 38 per cent, while in patients seen within 4 hours it is 27 per cent. In the present study the incidence was 21 per cent, and 53 per cent of patients were seen within 6 hours of onset. Further work is necessary to determine the incidence of ventricular fibrillation in relation to bradycardia in patients treated by a mobile ambulance service, since it is probable that the type of case seen by mobile intensive care units is different from that described here and from other coronary care units. In the present study, however, there was no evidence that arrhythmias were commoner in patients who had bradycardia within one hour of admission to hospital than in those in whom sinus bradycardia occurred later.

The present results suggest that atropine is not usually necessary for bradycardia at a rate of 50 to 60 a minute, though it can certainly relieve symptoms in patients who are faint and hypotensive with a heart rate of 40 a minute or less. It is our practice to use atropine cautiously in this situation and to recommend its use outside hospital in an initial dose of $0.3 \mathrm{mg}$ intravenously. In our experience a larger dose given quickly may cause tachycardia and increase chest pain. We do not regard bradycardia as a contraindication to the use of lignocaine, even though bradycardia in 
our experience has not often been associated with serious ventricular arrhythmias.

\section{References}

Adgey, A. A. J., Allen, J. D., Geddes, J. S., James, R. G. G., Webb, S. W., Zaidi, S. A., and Pantridge, J. F. (I97I). Acute phase of myocardial infarction. Lancet, 2, 5or.

Adgey, A. A. J., Geddes, J. S., Mulholland, H. C., Keegan, D. A. J., and Pantridge, J. F. (1968). Incidence, significance, and management of early bradyarrhythmia complicating acute myocardial infarction. Lancet, 2, 1097.

Briant, R. B., and Norris, R. M. (1970). Alprenolol in acute myocardial infarction: double blind trial. New Zealand Medical fournal, 71, 135.

Chapman, B. L. (197I). Prognostic factors in acute myocardial infarction treated in a coronary care unit. Australian and New Zealand Fournal of Medicine, $\mathbf{1}, 53$.

Gregory, J. J., and Grace, W. J. (1968). The management of sinus bradycardia, nodal rhythm and heart block for the prevention of cardiac arrest in acute myocardial infarction. Progress in Cardiovascular Diseases, 10, 505.

Han, J. (1969). Mechanisms of ventricular arrhythmias associated with myocardial infarction. American fournal of Cardiology, 24, 800.

Han, J., DeTraglia, J., Millet, D., and Moe, G. K. (1966a). Incidence of ectopic beats as a function of basic rate in the ventricle. American Heart fournal, 72, 632.

Han, J., Millet, D., Chizzonitti, B., and Moe, G. K. (I966b). Temporal dispersion of recovery of excitability in atrium and ventricle as a function of heart rate. American Heart fournal, 71, 481.

James, T. N. (1965). The chronotropic action of ATP and related compounds studied by direct perfusion of the sinus node. Fournal of Pharmacology and Experimental Therapeutics, 149, 233.
James, T. N. (1968). The coronary circulation and conduction system in acute myocardial infarction. Progress in Cardiovascular Diseases, 10, 410.

Jewitt, D. E., Balcon, R., Raftery, E. B., and Oram, S. (1967). Incidence and management of supraventricular arrhythmias after acute myocardial infarction. Lancet, 2, 734.

Lawrie, D. M., Greenwood, T. W., Goddard, M., Harvey, A. C., Donald, K. W., Julian, D. G., and Oliver, M. F. (1967). A coronary-care unit in the routine management of acute myocardial infarction. Lancet, 2, 109.

Lown, B., Fakhro, A. M., Hood, W. B., and Thorn, G. W. (1967). The coronary care unit. Fournal of the American Medical Association, 199, 188.

Norris, R. M. (I969a). Bradyarrhythmia after myocardial infarction. Letter to the Editor. Lancet, $\mathbf{I}$, 313.

Norris, R. M. (1969b). Heart block in posterior and anterior myocardial infarction. British Heart fournal, 31, 352.

Norris, R. M., Brandt, P. W. T., and Lee, A. J. (1969). Mortality in a coronary-care unit analysed by a new coronary prognostic index. Lancet, $\mathbf{I}, 278$.

Norris, R. M., and Croxson, M. S. (1970). Bundle branch block in acute myocardial infarction. American Heart fournal, 79, 728.

Norris, R. M., and Mercer, C. J. (1970). Sinus rate in acute myocardial infarction. Australasian Annals of Medicine, 19, 8IP.

Sowton, E., Leatham, A., and Carson, P. (1964). The Suppression of arrhythmias by artificial pacemaking. Lancet, 2, 1098.

Stock, E. (I97I). Cardiac slowing not cardiac irritability, the major problem in the pre-hospital phase of myocardial infarction. Australian and New Zealand fournal of Medicine, $\mathbf{1}, 315 \mathrm{P}$.

Requests for reprints to Dr. R. M. Norris, Green Lane Hospital, Green Lane West, Auckland, 3 New Zealand. 